\section{Cáncer sincrónico y metacrónico detectado con PET/CT en población oncológica}

\author{
DAVID LADRÓN DE GUEVARA', RODRIGO QUERA², \\ SEBASTIÁN ROZAS', SHMUEL SCHACHER, \\ JOSÉ MIGUEL REYES ${ }^{3}$, CLAUDIO PARDO ${ }^{1}$, RAÚL PEFAUR ${ }^{3}$
}

\section{Multiple primary malignant neoplasms detected by PET/CT in cancer patients}

Background: Imaging with F18-fluorodeoxyglucose PET/CT is used to determine sites of abnormal glucose metabolism and can be used to characterize and localize many types of tumors. Aim: To assess the prevalence of multiple primary malignant neoplasms (MPMN) detected by PET/CT in cancer patients. Material and Methods: F18-fluorodeoxyglucose PET/CT scans performed to 800 patients with a newly diagnosed cancer or with already treated tumors were retrospectively reviewed. In patients whose examination described incidental findings not related to the primary tumor, a research was done about further laboratory, imaging or pathological studies. Results: In 188 PET/CT scans (23\%) an incidental finding was found. Of these, 66 (35\%) were considered as MPMN, 12 as atypical metastases of a known primary tumor, 14 as false positive images (inflammatory or physiologic uptake) and 29 as benign or low grade tumors. In 67 cases (36\% of all incidental tumors), the finding was not confirmed. Seven percent of patients with a newly diagnosed tumor had a synchronic MPMN detected by PET/CT. Nine percent of patients with treated tumors developed a metachronous MPMN during their follow up. The most common incidental tumors were thyroid cancer in 15 cases, kidney cancer in 13, lung cancer in 10, colorectal carcinoma in 9, breast cancer in 6, prostate cancer in 4, non-Hodgkin lymphoma in 3 and pancreatic cancer in 2. Conclusions: A MPMN is detected by PET/CT in a significant number of cancer patients.

(Rev Med Chile 2017; 145: 1421-1428)

Key words: Neoplasms, Multiple Primary; Neoplasm, Second Primary; Positron-Emission Tomography.

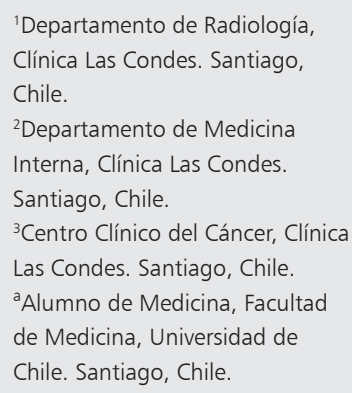

Recibidio el 15 de junio de 2017, aceptado el 24 de octubre de 2017.

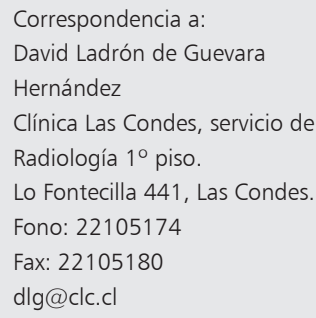

S e denomina neoplasias malignas primarias múltiples (NMPM) a dos o más tumores malignos distintos, que se generan en el mismo paciente. Según los criterios de Warren and Gates propuestos en $1930^{1}$, se requieren varias condiciones para calificar como NMPM: 1) que los tumores diagnosticados sean confirmados como de naturaleza maligna; 2) que sean histológicamente distintos, y 3 ) que se excluya que uno sea metástasis del otro. Esta definición se ha mante- nido vigente a pesar de su antigüedad, y estudios actuales sobre NMPM aún se basan en ella ${ }^{2}$.

Las NMPM pueden clasificarse según la temporalidad de aparición de las neoplasias malignas en: 1) Sincrónicas, si se detectan simultáneamente o hasta 6 meses del diagnóstico del primer tumor primario, o 2) Metacrónicas, si se detectan después de 6 meses de hecho el diagnóstico del primer tumor ${ }^{2,3}$.

La frecuencia de NMPM muestra una am- 
plia variabilidad en la literatura, de entre $0,7 \%$ a $11,7 \%$, dependiendo de si se basa en estudios de necropsias, de análisis de registros o bases de datos de pacientes oncológicos, o de seguimiento prospectivo de pacientes ${ }^{3}$. Su ocurrencia ha mostrado un alza sostenida en los últimos años, explicada tanto por la mejoría en los métodos diagnósticos, como por un alza real en la patología oncológica en una población cada vez más longeva ${ }^{2}$.

Lógicamente, la detección de NMPM estará supeditada a los métodos con que contamos para pesquisarla. El método de imagen ideal deberá poseer una alta sensibilidad y especificidad, adecuada relación costo/beneficio, disponibilidad e inocuidad, e idealmente que incluya la mayor cantidad de órganos posibles, o idealmente todo el cuerpo ${ }^{4}$. Uno de los grandes avances de este siglo en materia de imágenes médicas corresponde al advenimiento de las técnicas híbridas, siendo el PET/CT (Positron Emission Tomography/ Computed Tomography) lejos la más utilizada, particularmente en oncología 4 .

El PET/CT con F18-fluorodeoxiglucosa (F18FDG) tiene la capacidad de detectar lesiones que no son visibles con otros métodos de imagen, modificando el tratamiento de los pacientes en sobre $40 \%$ de los casos respecto la etapificación tradicional ${ }^{5}$. Esto es particularmente útil al querer detectar neoplasias insospechadas, las que son frecuentemente asintomáticas y corresponden generalmente a etapas precoces de la enfermedad y por lo tanto, potencialmente curables ${ }^{6,7}$.

Choi et $\mathrm{al}^{8}$ reportaron $4,8 \%$ de pacientes con una segunda neoplasia primaria detectada con PET/CT en pacientes oncológicos. Solo 59\% de las neoplasias fueron visibles con radiología convencional, es decir utilizando radiografías, ultrasonografía y TC.

Sin embargo, una de las debilidades de esta técnica es la alta ocurrencia de falsos positivos, dado principalmente por hipercaptación fisiológica de glucosa marcada en algunos órganos, y por procesos inflamatorios, especialmente de tipo granulomatoso, que pueden ser confundidos con actividad tumoral, y que muchas veces requerirán de un estudio dirigido adicional para su correcta interpretación ${ }^{4}$.

El objetivo de este trabajo es estimar la frecuencia de tumores sincrónicos y metacrónicos detectados con PET/CT en pacientes oncológicos, y determinar la naturaleza del resto de hallazgos incidentales que no fueron confirmados como malignidades incidentales.

\section{Pacientes y Método}

\section{Pacientes}

Se revisó la base de datos de PET/CT de nuestra institución, seleccionando los estudios PET/ CT corporales realizados con F18-FDG entre diciembre de 2008 y diciembre de 2013 que tuvieran antecedentes de neoplasia maligna confirmada por histología. La población oncológica seleccionada se dividió en dos grupos:

1. Etapificación: Pacientes con cáncer recientemente diagnosticado, sin tratamiento previo, que se realizaron el examen para etapificación de su neoplasia, y

2. Seguimiento: Pacientes con cáncer ya tratado en los que se solicitó PET/CT para re-etapificación o seguimiento alejado.

Los casos en que la patología neoplásica conocida no estuviera confirmada histológicamente fueron excluidos. Los estudios no oncológicos y aquellos oncológicos solicitados para evaluación de tratamiento (post quimioterapia o radioterapia, o interinos entre ciclos de quimioterapia) fueron excluidos.

Se revisó la ficha clínica y exámenes de todos los pacientes oncológicos seleccionados que presentaron un PET/CT con foco hipercaptante no típico de metástasis de su cáncer conocido, o una lesión sospechosa de un segundo tumor primario, en busca de una confirmación histológica de la lesión en cuestión. En caso de no existir estudio histológico o de ser éste no concluyente, se revisaron los exámenes de laboratorio (antígeno prostático específico, CEA, CA19-9), de imagen, endoscopias y PET/CT posteriores, que pudieran confirmar o descartar la sospecha de una segunda neoplasia concomitante.

\section{Adquisición y cuantificación del PET/CT}

Los PET/CT fueron realizados en un equipo GE Discovery STE de 16 canales, con adquisición de imágenes desde el vértex craneano hasta rodillas. Se cuantificó la captación de las lesiones en todos los casos, utilizando el índice SUVmax (Standarized uptake value maximum) . $^{9}$ 


\section{Tomografía computada}

Inmediatamente después de la adquisición del PET/CT y sin mover al paciente, se realizó una TC contrastada (TCc) de cerebro, cuello, tórax, abdomen y pelvis con protocolo y fases habituales, que incluyen fase arterial y portal para el abdomen, en todos los pacientes.

\section{Interpretación del examen híbrido}

El estudio PET/CT y la TCc fueron interpretadas en conjunto por tres radiólogos: un neuroradiólogo $(\mathrm{CP})$, un radiólogo sub-especialista de tórax (RP), y un doble especialista radiólogo/ médico nuclear (DLG), consensuando un informe único. Al interpretar el examen híbrido, se consideró lesión sospechosa de segunda neoplasia primaria: 1) foco hipercaptante de distribución no habitual para metástasis del primario conocido; 2) lesión con actividad metabólica distinta (mayor o menor) al tumor primario y sus metástasis; 3) lesión que aunque no fuera hipercaptante al PET, impresionaba como tumoral a la TC.

\section{Análisis de los datos}

Se consideró un "segundo tumor primario" aquel confirmado histológicamente como de histología maligna diferente a la neoplasia primaria que hizo consultar al paciente. Aquellas lesiones biopsiadas con resultado benignos, o descartadas por métodos invasivos (endoscopía, broncoscopía, laparoscopía) o bioquímicos (APE, marcadores tumorales) fueron catalogadas como "falsos positivos". Las lesiones sospechosas al PET/CT que no fueron biopsiadas ni seguidas fueron catalogadas como "no confirmadas".

\section{Definición de cáncer sincrónico y metacrónico}

Se definió cáncer sincrónico a aquel detectado al mismo tiempo que el cáncer conocido, o hasta 6 meses después de su diagnóstico, que demostró una histología distinta ${ }^{2,3}$. Se definió cáncer metacrónico a una segunda neoplasia maligna diagnosticada entre 6 meses y 10 años después del diagnóstico del primer cáncer ${ }^{2,3,10}$.

\section{Análisis estadístico}

Esencialmente es un estudio de prevalencia de tipo transversal, descriptivo, que no pretende estimar causalidad de las neoplasias, y no emplea estadística analítica. Sin embargo, se desea también estimar la positividad del PET/CT en la detección de hallazgos incidentales, estimando los verdaderos positivos y falsos positivos de la técnica, comparado a un gold standard que es la histología y/o seguimiento de las lesiones.

\section{Aspectos éticos}

La selección primaria de los pacientes se realizó utilizando la "base de datos PET/CT de CLC", aceptada por el Comité de Ética de Clínica Las Condes el día 1 de agosto de 2014. Todos los pacientes incluidos en la base de datos firmaron un consentimiento informado respecto a la realización del examen y a la utilización de sus imágenes de manera anónima para fines de investigación.

\section{Resultados}

De los 800 pacientes oncológicos estudiados, 403 correspondieron a individuos con cáncer recientemente diagnosticado, es decir en etapificación (mediana edad: 61 años, rango: 14-94 años), y 397 a pacientes con cáncer tratado en seguimiento (mediana edad: 60 años, rango: 8-88 años). La distribución según diagnóstico de referencia se muestra en la Tabla 1.

El 23,5\% de los PET/CT (188/800) mostró uno o varios hallazgos incidentales, de los cuales $66(35,1 \%)$ se confirmaron como una segunda neoplasia primaria (NMPM), 12 como metástasis de distribución atípica del primario conocido, 14 como falsos positivos del PET (inflamatorios, variantes) y 29 como tumores de bajo grado o benignos. En este último grupo figuran 14 meningiomas, 10 tumores mucinosos del páncreas, 2 adenomas paratiroídeos, 1 adenoma parotideo, 1 tumor pseudopapilar del pánceras, y 1 GIST (Gastrointestinal Stromal Tumor) gástrico de 18 $\mathrm{mm}$. En 35,6\% de los casos (67/188) el hallazgo no fue confirmado.

$\mathrm{Al}$ analizar el total de pacientes, 8,0\% (64/800) presentó una NMPM al PET/CT. Las neoplasias incidentales más frecuentes fueron cáncer tiroideo (15), carcinoma de células renales (13), cáncer pulmonar (10), cáncer de colon (9), cáncer de mama (6), cáncer de próstata (4), linfoma No Hodgkin (3), adenocarcinoma de páncreas (2).

\section{Pacientes en etapificación}

En el grupo de pacientes en etapificación se detectaron 30 neoplasias malignas sincrónicas en 28 
Cáncer sincrónico y metacrónico detectado con PET/CT - D. Ladrón de Guevara et al

Tabla 1. Tumores sincrónicos y metacrónicos encontrados incidentalmente con PET/CT en población oncológica, según diagnóstico de referencia

\begin{tabular}{|c|c|c|c|c|c|c|}
\hline \multirow{2}{*}{$\begin{array}{l}\text { Malignidad } \\
\text { conocida }\end{array}$} & \multicolumn{2}{|c|}{ Etapificación } & \multicolumn{2}{|c|}{ Seguimiento } & \multicolumn{2}{|r|}{ Total } \\
\hline & $\stackrel{\mathbf{n}}{\text { pacientes }}$ & $\begin{array}{c}2^{\circ} \text { primario } \\
\text { n pacientes }(\%)\end{array}$ & $\begin{array}{c}\mathbf{n} \\
\text { pacientes }\end{array}$ & $\begin{array}{c}2^{\circ} \text { primario } \\
\text { n pacientes }(\%)\end{array}$ & $\frac{\mathbf{n}}{\text { pacientes }}$ & $\begin{array}{c}2^{\circ} \text { primario } \\
\text { n pacientes }(\%)\end{array}$ \\
\hline Ca pulmonar & 143 & $8(5,6)$ & 47 & $3(6,4)$ & 190 & $11 \quad(5,8)$ \\
\hline LNH & 55 & $2(3,6)$ & 35 & $7(20,0)$ & 90 & $9(10,0)$ \\
\hline Melanoma & 28 & $2(7,1)$ & 23 & $1(4,3)$ & 51 & $3(5,9)$ \\
\hline Ca mama & 26 & $2(7,7)$ & 66 & $12(18,2)$ & 92 & $14(15,2)$ \\
\hline Ca colon & 24 & $3(12,5)$ & 52 & $2(3,8)$ & 76 & $5(6,6)$ \\
\hline Ca páncreas & 21 & 0 & 17 & $1 \quad(5,9)$ & 38 & $1(2,6)$ \\
\hline Ca gástrico & 20 & $2(10,0)$ & 12 & 0 & 32 & $2(6,3)$ \\
\hline Ca cabeza y cuello & 15 & $3(20,0)$ & 13 & $1 \quad(7,7)$ & 28 & $4(14,3)$ \\
\hline Enf. Hodgkin & 13 & 0 & 19 & $1 \quad(5,3)$ & 32 & $1(3,1)$ \\
\hline Ca recto & 11 & $2(18,2)$ & 14 & $2(14,3)$ & 25 & $4(16,0)$ \\
\hline Colangiocarcinoma & 6 & 0 & 7 & 0 & 13 & 0 \\
\hline Ca endometrio & 5 & 0 & 6 & 0 & 11 & 0 \\
\hline Ca vegiga-urotelio & 5 & 0 & 9 & $1(11,1)$ & 14 & $1(7,1)$ \\
\hline Ca cervico-uterino & 4 & 0 & 5 & 0 & 9 & 0 \\
\hline Otros & 27 & $4(14,8)$ & 72 & $5 \quad(6,9)$ & 99 & $9 \quad(9,1)$ \\
\hline Total & 403 & $28 \quad(6,9)$ & 397 & $36(9,1)$ & 800 & $64(8,0)$ \\
\hline
\end{tabular}

LNH: Linfoma no Hodgkin.

pacientes, correspondiendo a 7,0\% (28/403) de la muestra (Figura 1). Dos pacientes en etapificación tuvieron un doble tumor sincrónico además de la neoplasia conocida, es decir fueron portadores de tres neoplasias malignas simultáneas (Figura 2).

\section{Pacientes en seguimiento}

La mediana de tiempo entre el diagnóstico del primer cáncer y la detección de la neoplasia metacrónica por el PET/CT de seguimiento fue de 54 meses (rango: 7 meses-10 años). Se detec-

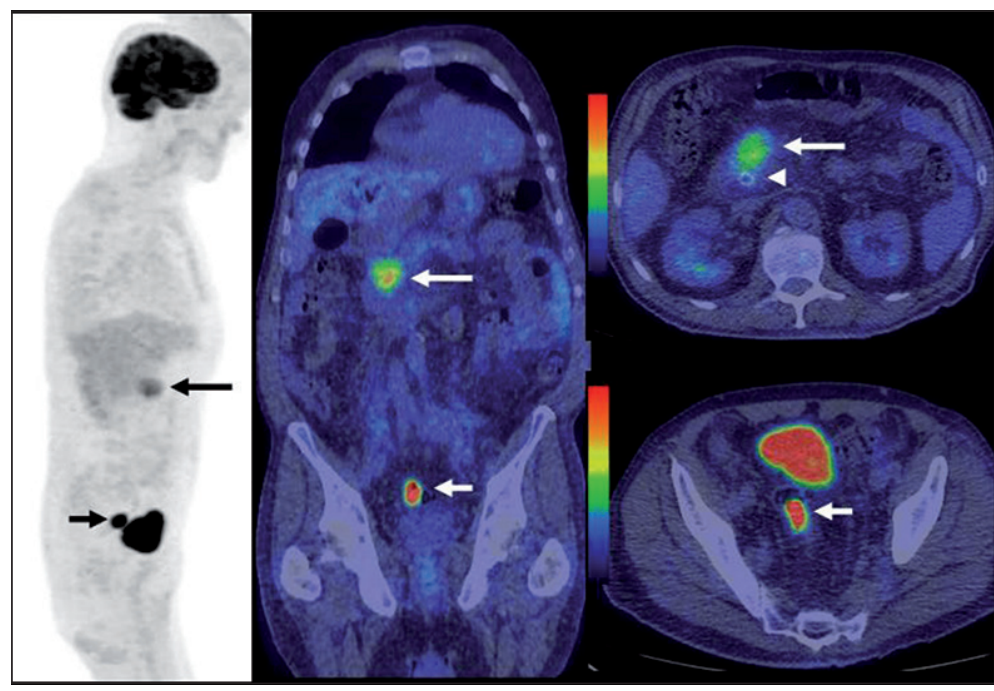

Figura 1. Paciente con cáncer de páncreas en etapificación. Se muestra una masa en cabeza pancreática con moderada captación de FDG (Flecha larga). Nótese la endoprótesis biliar adyacente a la masa (Cabeza de flecha). En el PET/ $\mathrm{CT}$ se pesquisa cáncer sincrónico de recto (Flecha corta), ávido de FDG. Por delante del tumor de recto se ve la vejiga normal con orina contrastada en su interior. 


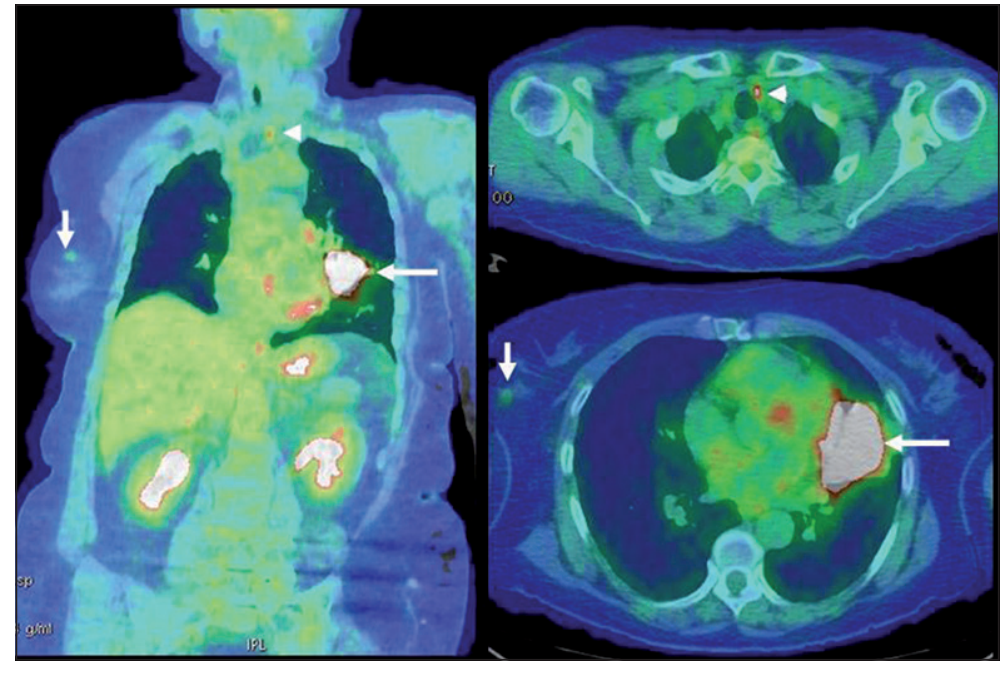

Figura 2. Paciente con cáncer pulmonar en etapificación. Masa parahiliar el LSI que resultó ser un carcinoma escamoso (flecha larga), el que se resecó completamente. EI PET/CT detectó dos neoplasias sincrónicas: un carcinoma de mama (flecha corta) y un carcinoma papilar de tiroides (cabeza de flecha).

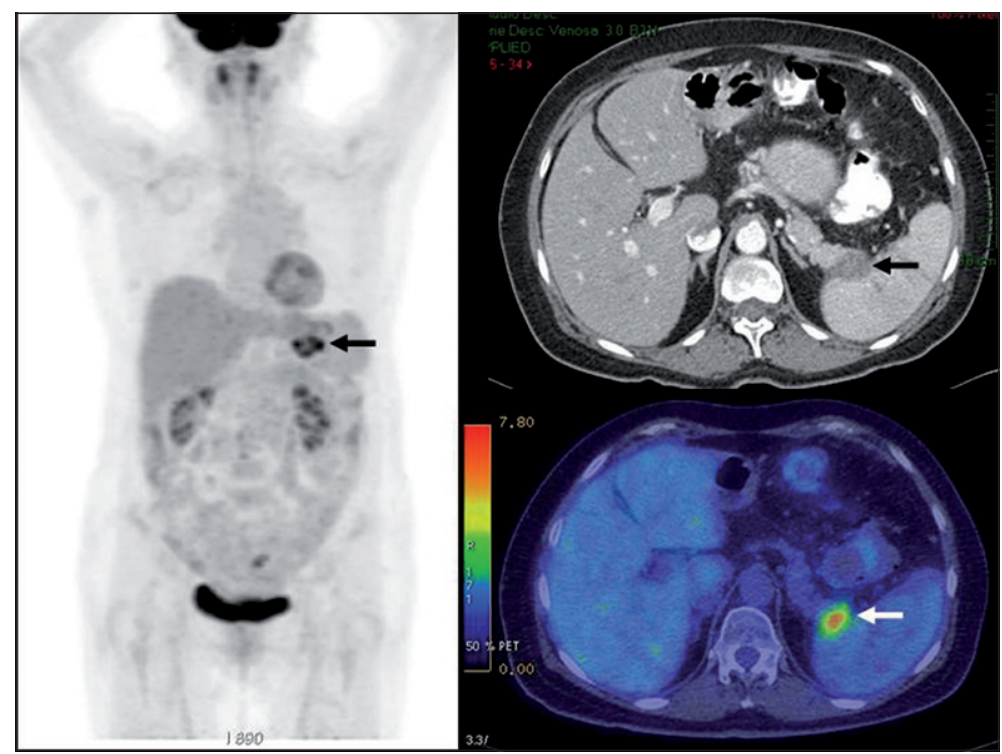

Figura 3. Paciente en control por cáncer de mama tratado hace tres años, donde el PET/CT detecta un adenocarcinoma pancreático metacrónico (flechas). taron 36 neoplasias metacrónicas en 36 pacientes, correspondiendo a 9,1\% (36/397) de la muestra (Figura 3).

\section{Discusión}

Diversas publicaciones han centrado su atención en la capacidad del PET de detectar neoplasias incidentales. Ya que la frecuencia de ocurrencia de neoplasias depende entre otras cosas de la pobla- ción en estudio, parece lógico separar dentro de la población oncológica a aquellos pacientes que debutan con un cáncer y que se realizan un PET de etapificación, de aquellos con una malignidad ya tratada y en seguimiento, ya que muchos de estos últimos han recibido terapias que pueden predisponer la aparición de nuevas neoplasias ${ }^{10-12}$.

En los pacientes con cáncer de reciente diagnóstico, la ocurrencia de neoplasias sincrónicas insospechadas reportada en la literatura fluctúa entre 1,7 y $17,6 \%$ de los individuos que se realizan 
un PET/CT corporal ${ }^{7,8,13-17}$. Sorprende el amplio rango expresado en la literatura, que puede deberse al tipo de pacientes en estudio, con menor incidencia en aquellos más jóvenes, sin noxas como el tabaco, alcohol, infecciones virales o bacterianas predisponentes, inmunosupresión, ni tratamientos previos (quimioterapia, radioterapia, hormonoterapia $)^{11,12}$. Además de factores ambientales y de estilo de vida, son importantes los factores genéticos y hereditarios que se sabe están relacionados con ciertas neoplasias, incluyendo diversos síndromes familiares o de ocurrencia esporádica $^{10,11}$. El 7,0\% de segundo cáncer sincrónico obtenido en nuestra muestra para una población oncológica general se encuentra en este rango, y representa un significativo porcentaje de pacientes cuyo manejo y pronóstico cambian notablemente al etapificar con PET/CT.

De acuerdo a las series publicadas, las neoplasias que más cáncer sincrónico presentan son las asociadas al tabaco, como el cáncer de cabeza y cuello (4-17\% con cáncer sincrónico $)^{17,18}$, cáncer pulmonar $(2,7-4,3 \%)^{19,20}$, cáncer esofágico $(5,5 \%)^{15}$, y cáncer gástrico $(5,4 \%)^{21}$. Nuestros resultados, si bien coinciden parcialmente, tienden a ser más ominosos, especialmente si se le compara a la literatura que se basa en la detección con métodos de imagen tradicionales, sin recurrir al PET/CT. Es sabido que el PET/CT aumenta la sensibilidad en la detección de tumores malignos respecto a la ecografía, radiología convencional y TC, por lo que es lógico que la frecuencia de tumores sincrónicos detectados sean mayores al utilizar dicha técnica híbrida ${ }^{7,8}$.

En nuestro medio hay escasos reportes de tumores sincrónicos/metacrónicos detectados con PET/CT, y con series pequeñas de pacientes ${ }^{19,22}$. Dos de ellas forman parte de evaluaciones preliminares de esta misma casuística, ahora ampliada a una población mayor. Nuestros reportes previos describen 4,3\% de neoplasias sincrónicas en pacientes con cáncer pulmonar recientemente diagnosticado ${ }^{19}$, y $5,9 \%$ de cáncer sincrónicos en PET/CT realizados a pacientes con cáncer de mama $^{22}$. Creemos que la actual casuística es un aporte en este sentido, ampliando la población en estudio, y permitiendo conocer la frecuencia de este fenómeno en población oncológica general y particular según tipo de cáncer.

El impacto clínico de encontrar otra neoplasia maligna en un paciente que ya es portador de un cáncer conocido debe ser cuidadosamente ponderado, evaluando las posibilidades terapéuticas de acuerdo a la naturaleza de las neoplasias en cuestión, principalmente de su grado de agresividad y avance de la enfermedad, y priorizando recursos y tiempo en aquellas de peor pronóstico. Debido a que la intensidad de hipercaptación de glucosa marcada se correlaciona directamente con el grado de desdiferenciación del tumor, y por lo tanto, con la agresividad del cáncer ${ }^{23,24}$, es más probable encontrar neoplasias mal diferenciadas y de curso agresivo con el PET, que son las que mayor captación presentan. Por otro lado, la pesquisa de malignidades en etapas evolutivas precoces, que son muchas veces asintomáticas, permite un tratamiento efectivo y mayor probabilidad de curación del cáncer ${ }^{7,8}$.

En el caso de pacientes que ya han sido tratados por un cáncer, la frecuencia de neoplasias metacrónicas reportada es mayor a la descrita para los pacientes con cáncer recientemente diagnosticado. Dong et al reportaron 8,5\% de frecuencia de una segunda neoplasia en el seguimiento de 633.964 pacientes tratados por otro cáncer ${ }^{25}$. Ueno et al describen 5,2\% de ocurrencia de una segunda neoplasia maligna en población oncológica, la mayoría dentro de los primeros 3 años de seguimiento $^{12}$. El 9\% obtenido en nuestra serie es similar a lo reportado en otros centros.

La ocurrencia de falsos positivos es una importante limitación del PET/CT, y se explica en parte por la baja especificidad intrínseca del método, ya que ciertos procesos inflamatorios, y aún fisiológicos, también pueden presentar captación de F18$\mathrm{FDG}^{24}$. Por este motivo, el examen puede resultar positivo en otras condiciones no neoplásicas, que muchas veces no tienen relevancia clínica, y en cuya investigación se consume tiempo y recursos. La utilización de la TC ayuda a disminuir este problema, ya que permite reconocer lesiones y diferenciarlas de situaciones fisiológicas o cambios inflamatorios ${ }^{26,27}$. Por otro lado, la TC puede detectar tumores de baja captación no visibles con $\mathrm{PET}^{4,28}$. Esto puede explicar la menor frecuencia de falsos positivos y mayor prevalencia de NMPM de nuestra casuística, comparada a otras publicaciones realizadas con PET no híbridos ${ }^{5,13,29}$.

Una de las limitaciones de nuestro estudio es el alto porcentaje de hallazgos del PET/CT que no fueron confirmados (35\%), lo que puede redundar en una subvaloración de la incidencia de tumores 
sincrónicos/metacrónicos. Sin embargo, comparativamente con otras series resulta un porcentaje relativamente bajo de lesiones "no confirmadas"29. Dicha falta de confirmación puede deberse en parte a la relativización de la importancia del hallazgo del PET/CT por priorización de recursos, especialmente en población con enfermedades avanzadas y en malas condiciones generales. Por otro lado, la pesquisa de una determinada lesión potencialmente maligna pero de baja agresividad (por ejemplo un cáncer tiroideo o prostático) no cambia mayormente el manejo y pronóstico de un paciente portador de un cáncer agresivo, resultando más razonable enfocar los recursos en tratar éste último.

\section{Conclusión}

El PET/CT detectó un segundo cáncer primario (NMPM) en $8,0 \%$ de los pacientes oncológicos. Este porcentaje es mayor en pacientes con antecedentes de cáncer tratado que se controlan con PET/CT (NMPM metacrónica), respecto a aquellos con neoplasias recientemente diagnosticadas (NMPM sincrónica). Un tercio de los incidentalomas del PET/CT se confirmó como NMPM, otro tercio mostró lesiones benignas o de bajo grado, y otro tercio no fue confirmado.

\section{Referencias}

1. Warren S, Gates O. Multiple primary malignant tumors: a survey of the literature and a statistical study. Am J Cancer 1932; 16: 1358-414.

2. Lv M, Zhang X, Shen Y, Wang F, Yang J, Wang B, et al. Clinical analysis and prognosis of synchronous and metachronous multiple primary malignant tumors. Medicine 2017; 96: 17(e6799).

3. Demandante CGN, Troyer DA, Miles TP. Multiple Primary Malignant Neoplasms. Case Report and a Comprehensive Review of the Literature. Am J Clin Oncol 2003; 26: 79-83.

4. Schoder H, Gonen M. Screening for Cancer with PET and PET/CT: Potential and Limitations. J Nucl Med 2007; 48: 4S-18S.

5. Hillner B, Siegel BA, Liu D, Shields AF, Gareen IF, Hanna L, et al. Impact of Positron Emission Tomography/Computed Tomography and Positron Emission Tomography (PET) Alone on Expected Management of Patients With Cancer: Initial Results From the National Oncologic PET Registry. J Clin Oncol 2008; 26: 2155-61.

6. Even-Sapir E, Lerman H, Gutman M, Lievshitz G, Zuriel L, Polliack A, et al. The presentation of malignant tumours and pre-malignant lesions incidentally found on PET-CT. Eur J Nucl Med Mol Imaging 2006; 33: 541-52.

7. Xu H, Zhang M, Zhai G, Li B. The clinical significance of 18F-FDG-PET/CT in early detection of second primary malignancy in cancer patients. J Cancer Res Clin Oncol 2010; 136: 1125-34.

8. Choi JY, Lee KS, Kwon OJ, Shim YM, Baek C-H, Park $\mathrm{K}$, et al. Improved Detection of Second Primary Cancer Using Integrated [18F] Fluorodeoxyglucose Positron Emission Tomography and Computed Tomography for Initial Tumor Staging. J Clin Oncol 2005; 23: 7654-9.

9. Wang G, Lau EWF, Shakher R, Rischin D, Ware RE, Hong E, et al. How Do Oncologists Deal With Incidental Abnormalities on Whole-Body Fluorine-18 Fluorodeoxyglucose PET/CT? Cancer 2007; 109: 117-24.

10. Tabuchi T, Ito Y, Ioka A, Miyashiro I, Tsukuma H. Incidence of metachronous second primary cancers in Osaka, Japan: Update of analyses using population-based cancer registry data. Cancer Sci 2012; 103: 1111-20.

11. Mehdi I, Shah AH, Moona MS, Verma K, Abussa A, Elramih R, et al. Synchronous and Metachronous Malignant Tumours expect the un-expected. JPMA 2010; 60: 905-9.

12. Ueno M, Muto T, Oya M, Ota H, Azekura K, Yamaguchi T. Multiple primary cancer: an experience at the Cancer Institute Hospital with special reference to colorectal cancer. Int J Clin Oncol 2003; 8: 162-7.

13. Agress H Jr, Cooper BZ. Detection of clinically unexpected malignant and premalignant tumors with whole-body FDG PET: histopathologic comparison. Radiology 2004; 230: 417-22.

14. Ladrón de Guevara D, Pefaur R, Pardo C. Neoplasias insospechadas detectadas en pacientes que se realizaron PET/CT de cuerpo entero. Rev Med Clin Condes 2010; 21: 845-50.

15. van Westreenen HL, Westerterp M, Jager PL, van Dullemen HM, Sloof GW, Comans EFI, et al. Synchronous Primary Neoplasms Detected on 18F-FDG PET in Staging of Patients with Esophageal Cancer. J Nucl Med 2005; 46: 1321-5.

16. Schwartz DL, Rajendran J, Yueh B, Coltrera M, Anzai Y, Krohn K, et al. Staging of Head and Neck Squamous Cell Cancer With Extended-Field FDG-PET. Arch Otolaryngol Head Neck Surg 2003; 129: 1173-8.

17. Stokkel MPM, Moons KGM, ten Broek F-W, van Rijk PP, Hordijk G-J. 18F-Fluorodeoxyglucose Dual-Head 
Positron Emission Tomography as a Procedure for Detecting Simultaneous Primary Tumors in Cases of Head and Neck Cancer. Cancer 1999; 86: 2370-7.

18. Kim SY, Roh J-L, Yeo N-K, Kim JS, Lee JH, Choi S-H, et al. Combined 18F-fluorodeoxyglucose-positron emission tomography and computed tomography as a primary screening method for detecting second primary cancers and distant metastases in patients with head and neck cancer. Ann Oncol 2007; 18: 1698-703.

19. Ladrón de Guevara D, Fuentes A, Fariña C, Corral C, Pefaur R. Valor pronóstico del PET/CT en cáncer pulmonar. Estudio de sobrevida y caracterización metabólica tumoral. Rev Med Chile 2013; 141: 41-8.

20. De Wever W, Vankan Y, Stroobants S, Verschakelen J. Detection of extrapulmonary lesions with integrated $\mathrm{PET} / \mathrm{CT}$ in the staging of lung cancer. Eur Respir J 2007; 29: 995-1002.

21. Nakamoto Y, Togashi K, Kaneta T, Fukuda H, Nakajima K, Kitajima K, et al. Clinical Value of Whole-body FDGPET for Recurrent Gastric Cancer: A Multicenter Study. Jpn J Clin Oncol 2009; 39: 297-302.

22. Ladrón de Guevara D, Guzmán P. Caracterización de enfermedad metastásica con PET/CT en cáncer de mama en etapificación y con recidiva postratamiento. Rev Chil Radiol 2013; 19: 21-8.

23. Dooms C, van Baardwijk A, Verbeken E, van Suylen RJ,
Stroobants S, De Ruysscher, et al. Association Between 18F-Fluoro-2-Deoxy-D-Glucose Uptake Values and Tumor Vitality: Prognostic Value of Positron Emission Tomography in Early-Stage Non-small Cell Lung Cancer. J Thorac Oncol 2009; 4: 822-8.

24. Jadvar H, Alavi A, Gambhir SS. 18F-FDG Uptake in Lung, Breast, and Colon Cancers: Molecular Biology Correlates and Disease Characterization. J Nucl Med 2009; 50: 1820-7.

25. Dong C, Hemminki K. Second primary neoplasms in 633,964 cancer patients in Sweden, 1958-1996. Int J Cancer 2001; 93: 155-61.

26. Metser U, Miller E, Lerman H, Even-Sapir E. Benign Nonphysiologic Lesions with Increased 18F-FDG Uptake on PET/CT: Characterization and Incidence. AJR 2007; 189: 1203-10.

27. Blake MA, Singh A, Setty BN, Slattery J, Kalra M, Maher MM, et al. Pearls and Pitfalls in Interpretation of Abdominal and Pelvic PET-CT. Radio Graphics 2006; 26: 1335-53.

28. Farwell MD, Pryma DA, Mankoff DA. PET/CT Imaging in Cancer: Current Applications and Future Directions. Cancer 2014; 22: 3433-45.

29. Ishimori T, Patel PV, Wahl RL. Detection of Unexpected Additional Primary Malignancies with PET/CT. J Nucl Med 2005; 46: 752-7. 